\title{
DISCURSO POR EL NOMBRAMIENTO DE SAN ALBERTO HURTADO COMO PATRONO DE LA FACULTAD DE DERECHO DE LA PONTIFICIA UNIVERSIDAD CATÓLICA DE CHILE
}

\author{
TEXT OF THE SPEECH GIVEN ON OCCASION OF ALBERTO HURTADO'S \\ APPOINTMENT AS "PONTIFICIA UNIVERSIDAD CATÓLICA DE CHILE'S" \\ LAW SCHOOL'S PATRON SAINT
}

\section{ARturo YrarráZaval Covarrubias ${ }^{*}$}

Hace 120 años, en 1888 , se fundaba la Universidad Católica de Chile cuando el Arzobispo de Santiago Mariano Casanova dicta el decreto de fundación. Este decreto del 21 de junio designaba a Joaquín Larraín Gandarillas, quien pasaría a ser el primer Rector, como presidente de la comisión promotora de la Universidad. En uno de sus considerandos se establecía que "si bien por el momento no se pueden establecer todas las Facultades, conviene comenzar con algunas y crear las restantes en la medida que se logre reunir los medios necesarios". La comisión resolvió colocar todos los trabajos de la Universidad bajo la protección del Divino Corazón de Jesús, crear una Sección Universitaria con un Curso de Leyes, también organizar un Internado Comercial para capacitar a jóvenes que trabajen en el comercio, bancos y oficinas fiscales y además organizar una Escuela Industrial para enseñar artes y oficios a niños pobres.

En la Asamblea General que se celebró el 8 de septiembre de 1888 el nuevo Rector plantea lo que deberá ser una Universidad Católica libre. Decía el Rector: "Una Universidad Católica es, en primer lugar, una vasta escuela en que se cultivan y enseñan las diferentes ramas del humano saber, en armonía con esas verdades fundamentales que ha puesto fuera de discusión la palabra infalible de Dios. Una

\footnotetext{
* Decano Facultad de Derecho de la Pontificia Universidad Católica de Chile, discurso pronunciado el 18 de agosto de 2008 con ocasión del nombramiento de San Alberto Hurtado como Patrono de la Facultad.
}

Universidad Católica es, además, un hermoso taller en que se educa al corazón y se forma el carácter de los jóvenes, y se les prepara para las diversas carreras y exigencias de la vida social. Una Universidad libre es, por fin, una Corporación que no vive del aliento ni de la inspiración oficial. La nuestra aspira al honor de deberlo todo a su propio y abnegado trabajo y a las simpatías que logren inspirar sus doctrinas, sus profesores y sus métodos... Estas grandes cosas encierran estas tres palabras: Universidad Católica libre".

Las clases comenzaron el 1 abril de 1889 con 50 alumnos que se matriculaban en el curso de Leyes y en el curso propedéutico de matemáticas. La Escuela de Leyes funcionó en un local en la calle Ahumada. El local se quemó dos años después y la Escuela tuvo que trasladarse a improvisados locales en la Alameda y, después, en Bandera. Fue el mismo Rector Larraín el que empezó a adquirir casas viejas situadas en la Alameda entre las calles Maestranza (ahora Portugal) y Lira. Esas casas viejas pasaron a ser la actual Casa Central. La evolución entre dicha fundación de la Facultad de Derecho y la actualidad ha sido bastante gradual y no exenta de grandes dificultades.

En marzo de 1918 se incorporaba Alberto Hurtado como alumno a nuestra Facultad, entrando por la misma puerta por la cual entramos nosotros todos los días. Era un joven alto, un poco desgarbado y de sonrisa fácil como son muchos de nuestros alumnos. Se vestía de riguroso terno oscuro como lo hacen nuestros alumnos para las pruebas solemnes y los exámenes orales. Nuestro santo se incorporaba a la Facultad más antigua de la Universidad y, según 
algunos historiadores, la más importante. Era 1918, el primer año en que todas las facultades de la Universidad Católica se ubicarían en la Casa Central, denominada presuntuosamente como el "Palacio Universitario".

Eran tiempos difíciles aquellos que le tocó vivir a nuestro Santo durante sus estudios universitarios. Terminaba en 1918 , la $1^{\text {a }}$ Guerra Mundial con 10 millones de muertos y daños materiales cuantiosísimos. Se firmaba en 1919 el Tratado de Versalles que imponía condiciones muy gravosas a los alemanes y que sería el principal caldo de cultivo utilizado por Adolf Hitler para llevar a Alemania a la Segunda Guerra Mundial. En Chile estábamos en pleno régimen parlamentario, con un inmovilismo generalizado, que luego del "ruido de sables", desembocó en una crisis institucional importante. Dentro del mundo cristiano todavía estremecía las conciencias la encíclica Rerum Novarum sobre la cuestión social.

Los años que Alberto Hurtado pasó en la Facultad serían vitales en la conformación de su personalidad. El no va a ser un estudiante más de Derecho. Trabajará en instituciones católicas de caridad, en las organizaciones estudiantiles, en política, en las batallas religiosas y en círculos de estudio. Además, era un muy buen alumno de Derecho. Todo lo haría impulsado por una gran vida interior. Es en la Universidad Católica donde comienzan a destacar los rasgos más atractivos de su personalidad.

Cuando Alberto Hurtado ingresó a estudiar Derecho, lo hizo en una universidad que tenía menos de 700 alumnos y solo tres Facultades: Derecho, Agronomía y Ciencias Físicas y Matemáticas. La Universidad no recibía fondos del Estado, no cobraba aranceles ni matrículas y dependía totalmente de la ayuda privada. Los títulos de la Universidad Católica no eran reconocidos por el Estado y los alumnos de Derecho debían rendir sus exámenes en la Facultad de Derecho de la Universidad de Chile. Por cierto que los profesores no recibían pago alguno por su docencia y no era fácil conseguirse profesores.

En el primer año de Derecho, Alberto tenía 54 compañeros de curso quienes tenían tres ramos: Filosofía del Derecho con Roberto Peragallo, Derecho Romano con Alberto
Cumming y Economía Política con Darío Urzúa. Fueron compañeros del santo Juan Gómez Millas, futuro gran Rector de la Universidad de Chile y dos veces Ministro de Educación, y Manuel Larraín, gran amigo suyo, futuro Vicerrector de la Universidad Católica y obispo, quien acuñó la famosa frase en el entierro del Padre Hurtado calificándolo como "una visita de Dios a nuestra patria". Recordando esos tiempos, escribirá el santo, tiempo después, lo siguiente: “ ¡con qué respeto se escribía entonces la palabra Ciencia y qué tremendo era ser encontrado en oposición a ella! El hombre entraba en el camino de la libertad ... La generación joven se lanzó con fiebre al estudio de ideas y sistemas. Lo que llamamos generación del 20 ... creía en la vida del pensamiento. Tenía hambre de lectura, de conferencias, de círculos de estudios".

Ese primer año de Derecho, el santo lo comenzó con un retiro predicado en Las Cruces por quien sería Rector dos años después, el ex alumno de Derecho monseñor Carlos Casanueva. De este retiro el santo escribió: "Cuando pienso con calma veo la necesidad de la perfección, lo siento con tal fuerza que debo buscarla... Y si no pongo todos los medios..., si no llevo una vida humilde y santa, qué responsabilidad la mía... ¿Qué cuenta daría a Dios de esas mil o más almas que Él esperaba salvar por mi medio, de esas Misas que dejaría de decir, de esa gloria que Él dejaría de recibir?”

Alberto Hurtado se incorpora, desde el comienzo de sus estudios en nuestra Facultad, a trabajar por los pobres en el Patronato de Andacollo. Los patronatos habían sido formados por la Iglesia Católica para dar ayuda a los más necesitados. Entre sus fundadores figuraban los entonces sacerdotes Carlos Casanueva y Martín Rücker (ambos rectores de la Universidad) y los profesores de Derecho Francisco de Borja Echeverría y Juan Enrique Concha. Se buscaba en los patronatos, el perfeccionamiento espiritual de los más necesitados y elevar su nivel de educación. El patronato de Andacollo incluía el río Mapocho y el barrio Mapocho que era uno de los más miserables de Santiago. Un amigo recordaba ese trabajo diciendo que Alberto "se acercaba a la gente pobre con alegría y con- 
fianza. Era hermoso contemplarlo tan integrado... cómo los consolaba, cómo los alentaba y cómo, en retorno recibía el cariño y la apertura de sus corazones".

En segundo año Alberto cursó, ahora con 40 compañeros en vez de los 55 originales, Derecho Constitucional con José María Cifuentes, Derecho Civil con Ezequías Alliende y Derecho Canónico con el sacerdote Clovis Montero. Desde el punto de vista espiritual, dado que monseñor Carlos Casanueva no había podido ser su director espiritual por lo ocupado que estaba en el Seminario de Santiago, el santo eligió al sacerdote de los Padres Franceses Damián Symon como su director hasta que entró al Seminario de la Compañía de Jesús. El padre Symon escribiría después sobre su dirigido lo siguiente: "Las virtudes que fueron aflorando y solidificándose fueron deslumbradoras, sobre todo lo que se refería a la caridad... No podía ver el dolor sin quererlo remediar. Tenía un corazón como un caldero en ebullición que necesita válvula de escape y aquí está la explicación de una multiformidad de obras de caridad".

En 1919 el santo se hizo el tiempo para seguir clases de latín que él pensaba sería muy útil si alguna vez lograba entrar al Seminario. Asimismo continuó en la Congregación Mariana, institución de piedad de los jesuitas en aquella época. Además, se integra a la Asociación Nacional de Estudiantes Católicos, entidad que había nacido en 1915 y que reunía a los jóvenes católicos. La Asociación había surgido como respuesta a la Federación de Estudiantes de Chile.

El santo se incorpora con entusiasmo a trabajar en las tardes en el Partido Conservador, donde llegó a ser, a muy temprana edad, su prosecretario. Alberto nunca mezcló su participación en política con la actividad gremial realizada dentro de la Universidad Católica ni con su permanente actividad social.

En tercer año ya quedaban solamente 30 de los que habían partido con Alberto los estudios de Derecho. Ese año 1920 se produjo una verdadera tormenta en la Universidad. Por conflictos con el arzobispo Errázuriz, renunció el Rector Martín Rücker y, junto con él, los Decanos de Derecho y Agronomía. Asume como rector un gran sacerdote, Carlos Casanueva. El Rectorado de don Carlos va desde 1920 hasta 1953 , y es un período en que la Universidad Católica se desarrolla en forma brillante. El Rector Casanueva dio desde el inicio de su Rectorado, una larga batalla por la autonomía de la Facultad de Derecho. Todos los años nuestros alumnos de Derecho debían rendir exámenes en la Universidad de Chile con una comisión compuesta por tres profesores de dicha Facultad. Después de mucho tiempo se autorizó al profesor del ramo de nuestra Facultad asistir al examen, pero solo con derecho a voz. No fue sino hasta 1953, el último año del Rectorado espectacular de Monseñor Casanueva, que se permitió mediante una modificación legal, gestionada por cierto por los profesores de la Universidad Católica, que la Facultad otorgase el título de Licenciado. El Rector diría en esos años de dependencia absoluta en cuanto a los exámenes y los cursos: "Nuestra Facultad de Leyes ha dado al Parlamento gran número de Diputados y Senadores, al Gobierno Ministros de Estado, diplomáticos y jefes de servicios públicos, a la Magistratura, a la Prensa, a los negocios, a la acción católica, hombres ya eminentes muchos de ellos que se imponen por sus méritos...” y no obstante lo anterior no contamos con ninguna autonomía universitaria.

Alberto tomó, en ese tercer año, Derecho Civil con Exequías Alliende, Derecho Internacional con Horacio Walker, Derecho Penal con Roberto Peragallo y Economía Social con Lorenzo Lobos. A mediados de año Alberto tuvo que suspender sus estudios para hacer el servicio militar. Se había producido un cambio de gobierno en Bolivia y el nuevo Presidente, junto al Presidente del Perú, plantearon una reivindicación de los territorios perdidos por ellos en la Guerra del Pacífico.

A cuarto año de Derecho pasaron 20 de los 55 alumnos originales y cursaron Derecho de Minas con Alejandro Lira, Derecho Comercial con Exequías Alliende, Derecho Procesal con Felipe Urzúa y Derecho Civil con Germán Riesco. Fue en cuarto año que Alberto escribió su Memoria para optar al grado de Bachiller en el tema "La reglamentación del trabajo de los menores". El tema era uno de 
los recomendados por Juan Enrique Concha, el profesor de Derecho de la Universidad Católica que constituía una de las grandes voces chilenas en el tema social. Por cierto, que el trabajo fue calificado de sobresaliente.

El último año de Derecho de Alberto encontraba a la Universidad Católica con los primeros frutos del brillante Rectorado de Monseñor Carlos Casanueva. Los alumnos, en dos años, habían aumentado de 700 a 1.200 y las unidades académicas eran ahora 8 en vez de 3: Derecho, Ingeniería, Politécnica, Agronomía, Arquitectura, Filosofía y Letras, Bellas Artes y Servicio Social. Alberto cursó en $5^{\circ}$ año Medicina Legal con Fernando Varas, Derecho Procesal con Felipe Urzúa, Hacienda Pública con José María Cifuentes y Derecho Administrativo con José Ramón Gutiérrez.

En 1923, ya egresado, Alberto presenta su Memoria final sobre "El trabajo a domicilio". Mientras Alberto estudiaba el examen de grado, fue durante todo el mes del Sagrado Corazón a la iglesia de los Padres Franceses en la Alameda. La razón era para pedirle a Dios que le solucionara los problemas económicos de su familia, para poder consagrarse totalmente al Señor. Los problemas económicos de la familia Hurtado Cruchaga se remontaban a 1905 cuando murió su padre y su madre viuda sacó a la venta el fundo de Los Perales de Tapihue. El comprador del fundo no pagó el precio, sin devolver el predio, alegando que había nulidad de la transacción. Fue el día del Sagrado Corazón que Alberto recibió un llamado informándole que se había llegado a una transacción con el demandado para el pago del precio adeudado.

Monseñor Casanueva al despedirlo con una misa en la Capilla de la Universidad, la misma Capilla nuestra de la Casa Central, decía de Alberto: "El se va, pero su ejemplo queda. El ejemplo del que ha sabido unir la profunda humildad con la fina valentía; la piedad acentuada con la risueña alegría; la energía con la mansedumbre; el ejemplo del que se siente rico en virtudes ha buscado siempre algún defecto para corregir en sí mismo y ha callado siempre los defectos del prójimo; del que ha tratado con la misma bondad al rico en su morada regia y al pobre en el conventillo; en una palabra, el ejemplo del que ha cumplido la ley amando a Dios sobre todas las cosas".

Alberto Hurtado parte a hacer sus estudios de filosofía y teología en el extranjero, especialmente en la Universidad Católica de Lovaina. A su regreso a Chile el rector Carlos Casanueva y su antiguo compañero de curso Manuel Larraín, Vicerrector Académico de la Universidad, realizan muchas gestiones para que enseñe en la Universidad. Monseñor Casanueva le decía en una carta en 1934: "Cuenta con que nuestra Universidad te ayudará para los viajes que hayas de hacer para cumplir dicha comisión, en la inteligencia que tú vendrás a desempeñar las clases de Pedagogía de esta Universidad, que son urgentísimas y de inmensa Gloria de Dios en las actuales circunstancias... ¡Si vieras qué esperanzas tengo cifradas en ti y cómo ansío, la hora de verte con nosotros realizándolas! Hay más campo que nunca, ni comparable con el que teníamos cuando eras estudiante".

Efectivamente Alberto Hurtado fue profesor de la Universidad Católica impartiendo asignaturas en distintas Facultades. Más relevante aún que su magnífica docencia, es lo que el santo escribió sobre la Universidad. Decía: "La Universidad debe ser el cerebro de un país, el centro donde se investiga, se planea, se discute cuanto dice relación al bien común de la nación y de la humanidad. Y el universitario debe llegar a adquirir la mística de que en el campo propio de su profesión no es solo un técnico, sino el obrero intelectual de un mundo mejor". Según nuestro santo la universidad ha de despertar en sus alumnos a lo menos las siguientes cualidades:

1. Sentido social, esto es, la conciencia que en el fondo ningún problema humano me puede ser extraño.

2. Sentido de responsabilidad social.

3. Sentido de escándalo, no para deshacerse en crítica estéril sino para remediar, para construir.

4. Hambre y sed de justicia.

5. Espíritu realizador. No quedarnos eternamente en las consideraciones generales por más hermosas que sean. Hacer. Es el tiempo de la acción. 
En Alberto Hurtado tiene gran influencia los escritos del Cardenal Newman sobre la Universidad, especialmente "The Idea of a University" (1852) y "University Teaching Considered in Nine Discourses" (1873). Desde estas fuentes se nutren parte de las críticas que el santo formula a la universidad de su época. Criticaba, siguiendo al cardenal Newman, la desconexión entre la producción intelectual y la realidad, el divorcio entre la teoría y la práctica y la ausencia de la relación entre fe y razón.

Del pensamiento del santo se concluyen tres grandes sugerencias para la universidad actual: conocimiento práctico, criterio social y formación de las personas. En cuanto al conocimiento práctico el santo planteaba que: "nos hace falta proponer soluciones concretas y precisas que aterricen de las grandes síntesis doctrinarias y se transformen en medidas prácticas bien estudiadas". Alberto le asigna una gran importancia a la investigación en el conocimiento práctico, así diría: "que por nada del mundo gastemos nuestras energías en críticas estériles, ni en repetir indefinidamente tesis generales que nadie niega. La visión del universitario es la del estudioso que traduce esos ideales grandes del hombre de la calle en soluciones técnicas, aplicables, realizables, bien pensadas. Hacerlo es la mayor obra de caridad que puede hacer un hombre, pues es la caridad social, pública”. La segunda sugerencia para la universidad actual se refiere al criterio social. Hablando a los alumnos de ingeniería de nuestra universidad les indicaba: "Nuestros alumnos, además de especialistas tienen una misión en la sociedad. El que se especializa demasiado, se aísla”. De acuerdo con el santo el criterio social se asegura mediante el estudio, difusión y aplicación de la Doctrina Social de la Iglesia. Su estudio lo recomendaba por cierto en todas las universidades católicas y también en las universidades laicas. La tercera gran sugerencia de Alberto se refiere a la formación de las personas en la universidad. En un retiro a profesores de la Universidad Católica les señalaba: "la Universidad ha de formar antes que todo, hombres. Hombres, no archivos ambulantes, ni grandes eruditos". Agregaba que el universitario debe ser un hombre culto, decía "esta actitud se adquiere no solo en una sola ciencia, sino que nuestro alumno habrá de cultivar diversas disciplinas". Asimismo, la formación del universitario debe incluir la relación de la fe con la razón, el hombre con Dios, con los demás y con lo creado. En Misión del Universitario, nuestro santo lo sintetiza magistralmente de la siguiente forma: "Producir la síntesis entre nuestras aspiraciones y nuestras posibilidades, entre un orden teórico y la capacidad de realizaciones".

Ahora bien, la Facultad de Derecho ha tenido durante bastante tiempo como su patrono a San Alfonso María de Ligorio. ¿Quién fue este santo patrono? Alfonso fue un gran santo italiano del siglo XVIII que a los 16 años era doctor en Derecho civil y canónico. Durante 14 años ejerció con particular brillo la profesión de abogado hasta que pierde su primer juicio. Alfonso defiende al duque Orsini di Gravina en contra del gran duque de Toscana. A pesar que Alfonso tenía toda la razón jurídica, el tribunal falla en su contra. Según algunos historiadores la Alta Corte habría sido sobornada y las fuertes presiones políticas habrían pesado más que el Derecho. Al salir de los palacios de justicia Alfonso dice: "Mundo, yo te conozco. ¡Adiós a los tribunales!”.

Además de brillante abogado Alfonso era un buen pintor y un excelente músico. Sin embargo, sus actividades más relevantes fuera del ejercicio profesional era su intensa vida de oración de a lo menos dos horas al día y atender a los enfermos del hospital de Nápoles, denominado por la gravedad de los enfermos, como los incurables. A los 27 años escucha el llamado de Dios: "Abandona el mundo y entrégate a mí", tras lo cual sale del hospital y corre a los pies de la Virgen María y después de una larga oración saca su espada de caballero y la deja sobre el altar. Durante su formación en el Seminario, Alfonso estudió en profundidad las obras de Santa Teresa de Avila, quien pasa a ser su "seconda Mamma" después de la Virgen María y las obras de San Francisco de Sales. Ya ordenado sacerdote trabaja intensamente con los más pobres de Nápoles sobre la base de capillas vespertinas que reunía en las noches después del trabajo a la gente pobre para hacerles verdaderos 
cristianos. Alfonso se dedica también a escribir sus obras, la mayoría de ellas en forma sencilla. Así se explica la enorme difusión de sus más de 111 obras con más de 20.000 ediciones y en más de 70 lenguas. Su obra monumental que principalmente le significó el título de doctor de la Iglesia fue su "Teología Moral", la que por primera vez unifica la moral de la Iglesia y la hace inclinarse hacia la benignidad. En esta obra examina más de 4.000 casos y con el apoyo de más de 60.0000 citas, encuentra solución para muchos problemas que pueden producirse en todas las circunstancias de la vida. Otra obra fundamental es Las Glorias de María, la obra que tuvo mayor difusión, de alrededor de 1.000 ediciones.

Se cuenta que cuando Alfonso estaba gravemente enfermo antes de morir, le leían pasajes de las Glorias de María y habiéndose olvidado que él las había escrito, exclamaba quién habrá escrito cosas de tan extraordinaria belleza. San Alfonso fue canonizado a mediados del Siglo XIX, pero fue en 1950 que Pío XII lo declaró patrono de los moralistas y confesores.

La Facultad de Derecho estuvo presente en la canonización de San Alberto Hurtado en Roma. En Santiago muchos de nuestros profesores, administrativos y alumnos siguieron la ceremonia por pantalla gigante instalada en la Casa Central. Fue emocionante cuando se descubrió la imagen de San Alberto en el frontis de San Pedro y nuestro querido Papa Benedicto XVI lo proclamó oficialmente como santo de la Iglesia entre aplausos enfervorizados de los que estábamos en la plaza y las banderas chilenas que anotaban la presencia de miles de compatriotas.

Posteriormente, la Facultad ha tenido Misas conmemorativas de San Alberto. En nuestra capilla de la Casa Central se ha incorporado un bonito retrato del santo cuando precisamente era alumno de nuestra Facultad. Por otra parte la Facultad, a través de generosas donaciones de sus profesores, alumnos y administrativos, hizo una preciosa escultura de Enrique Zamudio en que el santo aparece hablando en la Alameda sobre Cristo a personas de muy distintas condiciones.
Hoy en día, esta Facultad de Derecho tiene el inmenso honor de designar a san Alberto Hurtado como su patrono, además de san Alfonso María de Ligorio. Hemos esperado cumplir 120 años para tener dos patronos en la Facultad. Dos santos abogados que tuvieron las tribulaciones típicas de la santidad; dos ejemplos de vida espiritual; dos personas que hicieron de la caridad un verdadero apostolado, y dos grandes maestros que marcaron y dejaron huella en Italia y en Chile.

Para los profesores de nuestra Facultad, para todos nuestros alumnos y todos nuestros administrativos San Alfonso y San Alberto son verdaderos ejemplos a seguir, guiándonos en nuestras decisiones diarias.

Hemos querido iniciar nuestras celebraciones de los 120 años dando muchas gracias a Dios por esta fructífera existencia y para que nos dé muchas fuerzas en nuestras próximas actividades. El 26 de septiembre pretendemos tener una segunda celebración con un gran concierto del Mesías de Haendel. La tercera celebración será la inauguración de nuestro Edificio Derecho UC.

No podría terminar sin dar gracias a nuestro Gran Canciller y Cardenal, al Rector y las autoridades superiores de la Universidad, a los Decanos de nuestra Universidad, a los Decanos de otras Facultades de Derecho, a nuestros profesores, administrativos y alumnos y a todos quienes nos han apoyado en estas iniciativas en la Facultad.

En estos 120 años han sido cientos los profesores que enseñaron distintos cursos de la carrera con vocación notable y gran sacrificio. En estos 120 años han sido miles los alumnos que han pasado por nuestras aulas. La gran mayoría de nuestros ex alumnos se han destacado en el ejercicio profesional y en el servicio público. Hemos tenido Presidentes de la República, Cardenales y Obispos, Ministros de Estado, Senadores y Diputados, Rectores y Decanos de otras Universidades, profesores de Derecho, juristas destacadísimos y abogados en el ejercicio de la profesión, en la historia, en la filosofía, en la diplomacia, en el periodismo, en la educación y un gran santo Alberto Hurtado.

Por último un agradecimiento muy especial a todos los aportantes al nuevo Edificio 
Derecho y Espacio UC. Ellos nos permiten mirar con gran optimismo el futuro. Serán muchas nuevas generaciones las que podrán beneficiarse de esta gran infraestructura.

Por la intercesión de nuestros Patronos, San Alfonso y San Alberto, imploramos la protección del Sagrado Corazón de Jesús para
Nuestra Facultad así como le encomendamos su futuro y su labor de formación humana, académica y profesional.

¡Que el Señor bendiga a todos!

Por Dios, la Patria y la Universidad Católica de Chile. 